\title{
The cell biology of embryonic stem cells
}

\author{
Duanqing Pei ${ }^{1}$ \\ ${ }^{1}$ Center for Stem Cell Biology and Regenerative Medicine, Guangzhou Institute of Biomedicine and Health, Chinese Academy of \\ Sciences, Guangzhou 510663, China
}

The hallmark features of embryonic stem cells are the capacity to undergo limitless self-renewal and differentiate into virtually any cell type of our body. These two properties establish the unique role of ES cells in the field of regenerative medicine in both research and clinical applications. First of all, stem cells and their derivatives are important tools in biomedical research, providing informative models for discovering the function of novel genes and establishing novel drug screens in the postgenomic era. On the other hand, regenerative medicine based on stem cell technology may become the third therapeutical modality in addition to the well established classic therapeutic modalities of chemo- and surgical therapies, providing much needed clinical solutions to many degenerative diseases such as Parkinson diseases. However, the molecular mechanisms that govern the selfrenewal and differentiation of embryonic stem cells remain largely unclear, thus hampering the ultimate application of embryonic stem cells in biomedical research and regenerative medicine. We have been focusing on three core transcription factors Oct4/ Sox2/Nanog in maintaining stem cell pluripotency. Interestingly, these factors have been demonstrated to behave like oncogenes in the non-stem cell context. Our recent finding suggest that each member of this core family of factors perform different roles in mediating the transcription of downstream genes and cooperate to ensure that ES are pluripotent under a wide range of culture conditions. A paradigm shift is underway in stem cell research and cell biology may be leading such shift in the coming decades.

Cell Research (2008) 18:s6. doi: 10.1038/cr.2008.96; published online 4 August 2008

Correspondence: Duanqing Pei

E-mail: pei_duanqing@gibh.ac.cn

Duanqing Pei, PhD, is currently Professor and Deputy Director General at Guangzhou Institute of Biomedicine and Health (GIBH), Chinese Academy of Sciences. He received his PhD from Penn in 1991.During this period of time, he specialized in MMP biology. Upon returning to China, he initiated studies aimed at the elucidation of mechanisms governing embryonic stem cell self renewal and pluripotency. Through works on Oct4, Sox2, Nanog and FoxD3, Dr Pei and his colleagues have established several working models and research platforms for pluripotency and somatic cell reprogramming. Nationally, Dr Pei serves as a member of the Expert Committee for the National Key Research Program "Reproduction and Development" and a council member of the 863 High-Tech Program in Biomedicine. 